\title{
Determinantes del balance laboral y familiar en Monterrey, México
}

\section{Determinants of work and family balance in Monterrey, Mexico}

Karina Elizabeth Delgado-Ledezma`

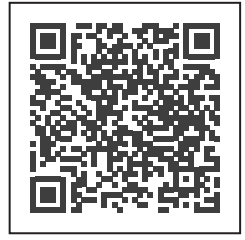

Articulo de investigación:

Fecha de recepción:

05/12/2019

Fecha de aceptación:

21/10/2020

Esta publicación se encuentra bajo licencia:

Creative Commons

Reconocimiento-

NoComercial-

SinObraDerivada 4.0

Internacional

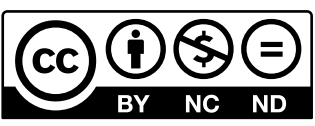

\section{Resumen}

El presente artículo analiza los determinantes que intervienen en el balance laboral y familiar en el municipio de Monterrey en el Estado de Nuevo León, México. Diversos estudios recientes señalan que dicho balance es crítico para poder lograr un equilibrio en el individuo. Por consiguiente, asociaciones civiles e instituciones mexicanas han tenido un impacto en el desarrollo de las políticas públicas sobre el balance laboral y familiar, que ha derivado en modificaciones de estatutos y leyes de diferentes países con un enfoque en la armonía entre el trabajo y la familia. Por lo tanto, el objetivo del artículo es cuantificar los determinantes que inciden en el balance laboral y familiar. Específicamente, se pretende analizar el efecto de la responsabilidad social empresarial, el estrés laboral, los incentivos fiscales, las políticas públicas y la flexibilidad de horario en el balance laboral y familiar. En este estudio se entrevista a 103 empleados y por medio de la técnica de análisis factorial se encuentra que la responsabilidad social de la empresa donde trabajan, así como el estrés laboral tienen un impacto directo en la variable dependiente, mientras que la práctica de las variables de incentivos fiscales, políticas públicas y flexibilidad de horario presentan un impacto no significativo.

Palabras clave: balance laboral, equilibrio familiar, estrés laboral, análisis factorial, caso de estudio, México.

1 Maestra en Administración de Empresas, Facultad de Contaduría Pública y Administración, Universidad Autónoma de Nuevo León, México. cpkarina. delgado@gmail.com, ORCID: https://orcid.org/0000-0002-5742-7641 


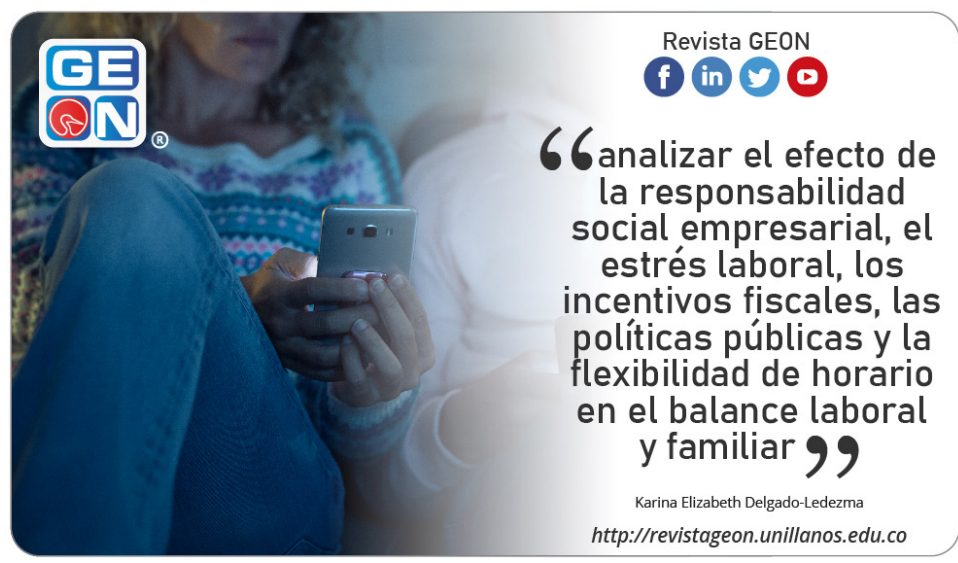

Códigos JEL: M12 Gestión de personal; Ejecutivos; Compensacion Ejecutiva; Economía del personal, M50 Gestión laboral, M54 Métodos de clasificación, C38 Análisis de conglomerados Componentes principales Modelos de factores

\section{Abstract}

This article analyzes the determinants involved in the work and family balance in the municipality of Monterrey in the state of Nuevo León, Mexico. Several recent studies indicate that such balance is critical for an employee. Thus, non-profit organizations and Mexican institutions have had an impact on the development of public policies about work and family balance, leading to new amendments in constitutional laws across different countries. In that sense, the objective of this article is the quantification of the factors that impact on work and family balance. Specifically, social responsibility, work stress, tax incentives, public policies, and work

Cómo citar este artículo / Toreference this article:

Delgado-Ledezma, K. E. (2021). Determinantes del balance laboral y familiar en Monterrey, México. Revista GEON (Gestión, Organizaciones Y Negocios), 8(1), 1-12. https://doi. org/10.22579/23463910.203 schedule flexibility were analyzed. In this study, the factor analysis technique included data from a sample of 103 employees. Results indicated that both a firm's social responsibility and work stress have a direct impact on the work and family balance. In the other hand, public policies and work schedule flexibility had a non-significant impact. 
Cómo citar este artículo / Toreference this article:

Delgado-Ledezma, K. E. (2021). Determinantes del balance laboral y familiar en Monterrey, México. Revista GEON (Gestión, Organizaciones Y Negocios), 8(1), 1-12. https://doi. org/10.22579/23463910.203
Keywords: Work balance; Family balance; Stress at work; Factor analysis; Case study; Mexico.

Code JEL: M12 Personnel Management Executives Executive Compensation, M50 Personnel Economics; M54 labor Management; C38 Classification Methods; Cluster Analysis; Principal Components; Factor Models

\section{Introducción}

El balance laboral y familiar implica prácticas que inicialmente se desarrollaron en países europeos. Sin embargo, se ha empezado a analizar la implementación de dichas prácticas en centros de trabajo en México. Algunas de estas incluyen horarios flexibles, permisos para asistir a asambleas escolares, estudiar algún posgrado sin necesidad de reponer tiempo o que descuenten parte del sueldo, aprovechamiento de banco de horas, entre muchas otras.

En la última década, organismos no gubernamentales, entidades e instituciones mexicanas han influido en la generación de políticas públicas relacionadas con el balance laboral y familiar. La base de la argumentación ha provenido de organismos internacionales como las Naciones Unidas, que derivó en las modificaciones legislativas a favor de dicho balance. Cabe señalar que en el año 2018 se estableció por decreto el 1 de junio como el Día Nacional del Balance Trabajo-Familia en México.
De acuerdo con Poll (2013), generaciones como la de los millenials muestran una alta importancia por la obtención de beneficios y equilibrio en la vida laboral y familiar. Esto ha llevado a las empresas a cambiar sus esquemas hacia el cumplimiento por objetivos y el desarrollo de conocimiento en las necesidades y desarrollo personal de sus trabajadores. Por tanto, han obtenido beneficios en sus indicadores de productividad por la existencia de un clima organizacional propicio para los trabajadores.

No obstante, dichos avances se presentan principalmente en el ámbito privado, a partir de prácticas voluntarias, de buena fe y sin beneficios fiscales. Más aún, según la Organización para la Cooperación y el Desarrollo Económico (OCDE), México es el penúltimo país en el que los empleados cuentan con tiempo libre. Por tanto, el objetivo del presente artículo es la cuantificación del efecto de la responsabilidad social empresarial, el estrés laboral, los incentivos fiscales, las políticas públicas y la flexibilidad de horario en el balance laboral y familiar. 


\section{Contexto teórico}

El balance laboral y familiar ha tenido un gran interés para los estudiosos de la calidad de vida en el trabajo. Desde la Revolución Industrial, una de las principales preocupaciones ha sido la explotación infantil, mientras que en los períodos de recesión la falta de empleo y sus consecuencias han sido el foco de atención. Actualmente, la discusión se ha centrado en las demandas excesivas de trabajo de entornos en economías tanto desarrolladas como en desarrollo (Guest, 2002).

Se han iniciado líneas de investigación orientadas hacia el beneficio que las empresas pueden obtener al integrar el balance laboral y familiar como una herramienta del departamento de recursos humanos para motivar a los trabajadores (Felstead et al., 2002). El objetivo que tienen las líneas de investigación del balance laboral y familiar es alinear el área profesional y no profesional en la vida del trabajador y de esta manera reducir el conflicto entre el trabajo y la familia. Los resultados de este planteamiento se pueden vincular con la mejora en la salud de los trabajadores (Frone y Yardley, 1996), así como en su relación familiar y productividad laboral (Howson y O'Driscoll, 1996).

De esta forma, la práctica de balance laboral y familiar podría mejorar el desempeño financiero de las empresas según Allen (2001), aunque los datos empíricos que respaldan estas afirmaciones son escasos. Aunado a esto, la armonía que pueda tener el trabajo y la vida personal del trabajador de for- ma equilibrada mejora la calidad de vida de este (Chan et al., 2016).

Más aún, según Leung et al. (2019), el apoyo emocional de la familia es un recurso valioso para el trabajo. De acuerdo con Sirgy y Lee (2017), existen beneficios del balance trabajo-familia desde una perspectiva individual del empleado (satisfacción laboral/ marital), como una perspectiva organizacional (i.e., ausentismo, rotación). Asimismo, según Brough y O'Driscoll (2010), existen beneficios relacionados con resultados positivos a nivel personal y organizacional, como lo son la satisfacción en el trabajo, en la familia y la mejora en el desempeño.

De acuerdo con lo planteado por Guest (2002), existen tres líneas de influencia que impulsan el debate en el balance laboral y familiar. La primera se centra en el tipo de trabajo que afecta al empleado; la segunda implica la vida personal del trabajador ante la falta de balance con el trabajo; finalmente, la tercera se centra en los individuos y las consecuencias del desbalance trabajo-familia. De acuerdo con Hirschi et al. (2019), el ser humano se adapta a las condiciones de su entorno mediante la modificación o el abandono de las metas sobre trabajo o familia ante una falta de equilibrio. Una de las razones que se enumeran para establecer políticas de balance laboral y familiar es el facilitar la integración de las mujeres al trabajo, esto para ayudar a cubrir la demanda laboral.

Según Edwards y Rothbard (2000), las exigencias laborales pueden derivar 
en conflictos familiares. Cabe señalar que Haar (2013) encontró diversos efectos psicológicos como el cansancio emocional, la ansiedad y la depresión por la falta de balance entre el trabajo y la vida familiar. Aunado a lo anterior, Marks y McDermid (2016) señalan que un trabajador puede experimentar un rol negativo, que deriva en la falta de involucramiento en el desempeño de ambos roles, y llegar a expresar apatía y cinismo. Finalmente, Greenhaus et al. (2003) señalan que un balance negativo se puede percibir como una falta de involucramiento y aislamiento del trabajador.

Como componente del balance laboral y familiar, la flexibilidad laboral ha emergido en la década de los 90 como una respuesta ante la rigidez del mercado laboral. Según White et al. (2003), dicha flexibilidad se produjo como un elemento atenuador de las protestas sindicales y el ajuste de sueldos en épocas de recesión. En el caso de las mujeres trabajadoras, la flexibilidad laboral fungió como un mecanismo para reclutar y retener el talento al promover entornos de trabajo familiarmente amigables. Por consiguiente, se abordó en países occidentales la fragilidad macroeconómica en el sector empresarial a partir de la contratación de mujeres en esquemas de medio tiempo. Además de lo anterior, el entorno social actual ha modificado su composición con respecto a la dinámica familiar (Eby et al., 2005; Jiménez et al., 2009). Los hogares en los que existe más de un ingreso y la cantidad de mujeres que se dedican a otras actividades adicionales al hogar son actualmente cada vez más comunes (Chioda, 2011). De igual manera, las mujeres han tenido grandes e importantes avances el ámbito político. Es en este sentido que el propósito de este artículo es cuantificar el impacto que trae consigo los determinantes expuestos por Boyer, en un caso de estudio realizado en el municipio de Monterrey en México.

Se decidió tomar en consideración el caso de Monterrey, en el Estado de Nuevo León, México, debido a la gran actividad e incidencia que se ha tenido por parte de la sociedad civil, la cual, desde el año 2016, estuvo promoviendo la iniciativa del Día Nacional del Balance Trabajo-Familia, inicialmente en Nuevo León y posteriormente llevándolo a todo México. Lo anterior mediante la implicación de un trabajo de cabildeo, el establecimiento de alianzas con actores estratégicos, la sensibilización, la visibilidad de la realidad, los retos y las buenas prácticas que viven las y los trabajadores en su día a día. La iniciativa tuvo sus inicios en el año 2015 con la Asociación Nacional Cívica Femenina, AC (ANCIFEM), la cual realizó un análisis exploratorio del impacto y el costo que tendría para las empresas y sus trabajadores, donde encontraron altos índices de estrés, insatisfacción, bajo desempeño laboral, ausentismo y rotación. Por lo tanto, se decidió incidir en la cultura laboral, en donde el empleado es el centro y el trabajo un medio para el desarrollo integral del individuo.

Posteriormente, la asociación incluyó 3 objetivos: 1. Posicionar la cultura del balance trabajo-familia en la agenda local y la opinión pública; 2. 
Impulsar que cada 1 de junio se celebre en Nuevo León y México el Día del Balance Trabajo-Familia (Día BTF); y 3. Promover el diálogo y las propuestas para que más centros de trabajo incluyan prácticas que permitan balancear la vida personal y familiar con la laboral. Lo anterior alineado con 3 de los 17 objetivos de desarrollo sostenible de la Organización de las Naciones Unidas, los cuales son: 5 igualdad de género; 8 trabajo decente y crecimiento económico y 17 alianzas para lograr los objetivos (ONU, 2020).

Finalmente, el 1 de junio de 2016 se entregó al congreso del Estado de Nuevo León la iniciativa, que dio pie al cabildeo y al trabajo en el interior del recinto legislativo y tuvo como resultado la aprobación, en el congreso local, del decreto para la celebración del 1 de junio como el Día del Balance Trabajo-Familia en Nuevo León y en 2019 para todo el país (Diario Oficial de la Federación, 2018).

\section{Materiales y métodos}

En esta investigación se estableció un cuestionario como instrumento para la recolección de datos durante la entrevista a los empleados de diferentes empresas, tanto públicas como privadas, localizadas en el municipio de Monterrey en Nuevo León, México. El proceso que se siguió fue a través de un muestreo no probabilístico de tipo bola de nieve, que nos dio la oportunidad de recabar la información que se necesitaba de los sujetos de estudio, siempre y cuando fueran recomendados de boca en boca para cubrir el perfil deseado; es decir, que fueran personas que estuvieran más de dos años laborando en Monterrey y que tuvieran más de 18 años.

La encuesta se dividió en dos apartados: el primer bloque capturó las características individuales de los empleados, tales como: edad, escolaridad, puesto que desempeña, estado civil, ingreso, tipo de empresa, cargo y antigüedad. En el segundo bloque se preguntó sobre la percepción que los empleados tienen acerca de la relación trabajo-familia; específicamente se trató de cuantificar el efecto de la responsabilidad social empresarial, el estrés laboral, los incentivos fiscales, las políticas públicas y la flexibilidad de horario en el balance laboral y familiar en trabajadores de empresas en Monterrey, Nuevo León. Este último apartado se realizó con base en la escala tipo Likert, con el fin de proporcionarnos una mayor facilidad y evaluar el balance familia y trabajo que existen en los empleados de empresas en la región objeto de estudio, siendo la escala: 1 = totalmente en desacuerdo, 2 = en desacuerdo, $3=$ neutral, 4 = de acuerdo y $5=$ totalmente de acuerdo.

El instrumento aplicado en el presente estudio fue validado por expertos en el tema. Asimismo, se midió el grado fiabilidad con el Alfa de Cronbach, que dio como resultado 0,824.

La población considerada para este estudio fueron los empleados de empresas públicas y privadas, las cuales se describen en la siguiente sección. Se recabó un total de 103 trabajadores, que estuvieron dispuestos a contestar el instrumento. 
Es conveniente mencionar que en este estudio solo se analizaron los dos bloques del cuestionario: en la primera parte del instrumento se llevó a cabo un análisis descriptivo de las variables de control (edad, escolaridad, puesto que desempeña, estado civil, ingreso, tipo de empresa, cargo y antigüedad), mientras que en el segundo bloque se aplicó la técnica de análisis factorial con la finalidad de confirmar los constructos o dimensiones propuestos por Boyer et al. (2005).

\section{Resultados}

De acuerdo con la composición de las variables derivadas del análisis factorial (véase la tabla 4 más adelante), el primer grupo de factores se relaciona con la dimensión definida como responsabilidad social empresarial, que implica cómo se sienten los entrevistados en su lugar de trabajo. El segundo grupo puede ser clasificado como aquellos factores que producen estrés laboral. En esta categoría se resalta cómo las personas perciben en su trabajo un ambiente tenso y con mucha carga profesional. El tercer grupo engloba la categoría sobre la percepción de los incentivos fiscales; se encuentra que la mayoría de los entrevistados considera que debería haber un incentivo en este rubro para encontrar mayor equilibrio entre trabajo y familia. El cuarto grupo comprende la implementación de las políticas públicas, donde las personas consideran que el buen uso e implementación de políticas laborales ayudarían a encontrar un equilibrio entre el mercado laboral y personal. Por último, el quinto grupo, denominado flexibilidad de horarios, establece que si existiera un horario flexible en sus respectivos trabajos, estos traerían una mayor estabilidad o equilibrio laboral y familiar.

La tabla 1 muestra el análisis descriptivo de las variables de control. Se aprecia que el número de encuestas realizadas en la investigación es un total de 103 empleados, de las cuales se destaca que la mayoría de los participantes es del sexo masculino y que la edad promedio de los encuestados se encuentra en 39,3 años. En cuanto al estado civil, la mayoría se encuentra soltero. Asimismo, la mayoría argumenta no tener hijos.

Tabla 1.Datos generales del encuestado

\begin{tabular}{lcc}
\multicolumn{1}{c}{ Sexo } & Frecuencia & Porcentaje \\
\hline Femenino & 46 & 44,7 \\
\hline Masculino & 57 & 55,3 \\
\hline Total & 103 & 100 \\
\hline Edad & & \\
\hline De 25 a 34 & 34 & 33 \\
\hline De 35 a 44 & 47 & 46 \\
\hline De 45 a 54 & 11 & 11 \\
\hline De 55 a 64 & 6 & 6 \\
\hline De 65 a 74 & 5 & 5 \\
\hline Total & 103 & 100 \\
\hline Estado civil & & \\
\hline Soltero & 66 & 64,1 \\
\hline Casado & 31 & 30,1 \\
\hline Divorciado & 3 & 2,9 \\
\hline Viudo & 3 & 2,9 \\
\hline Total & 103 & 100 \\
\hline Número de hijos & & \\
\hline Cero & 72 & 69,9 \\
\hline Uno & 5 & 4,9 \\
\hline Dos & 16 & 15,5 \\
\hline Tres & 6 & 5,8 \\
\hline Cuatro & 4 & 3,9 \\
Total & 103 & 100 \\
\hline Euente: & &
\end{tabular}

Fuente: elaboración propia. 
El análisis presentado implicó la utilización de la técnica de análisis factorial, la cual tiene como objetivo la cuantificación de la percepción actual que tienen los empleados que laboran en el municipio de Monterrey en Nuevo León respecto al balance laboral y familia, pues creemos que parte de la percepción del balance familiar y trabajo está relacionada de manera directa con factores de responsabilidad social empresarial, estrés laboral, incentivos fiscales y políticas públicas. Mediante dicho análisis, se determinaron las agrupaciones de variables por medio de las preguntas que las representan, para así tener la posibilidad de realizar la explicación de grupos a partir de un número reducido de variables. De esta forma, se derivó en una mejor interpretación de la variabilidad existente entre estas.

En la tabla 2 se muestra el tipo de lugar donde se labora, siendo la empresa el sitio de mayor frecuencia. Con respecto a la antigüedad de la empresa, la mayoría se encuentra en los 7 años, y 27 del total de los encuestados tienen un nivel de puesto de coordinación, con un ingreso mensual entre los 10.000 y los 20.000 pesos, con un tiempo de traslado de máximo 30 minutos.

En la tabla 3 se especifican los promedios y las desviaciones estándar para cada uno de las interrogantes. En dicho sentido, la pregunta con menor promedio fue la P20, mientras que la P17 fue la más alta. Cabe señalar que se obtuvo un promedio de 3,91 de la escala total, así como un valor de 0,89 para la desviación estándar.
Tabla 2.Datos laborales del encuestado

\begin{tabular}{|c|c|c|}
\hline Concepto & Frecuencia & Porcentaje \\
\hline \multicolumn{3}{|c|}{ Tipo de empresa donde labora } \\
\hline Gubernamental & 18 & 17,5 \\
\hline Pública & 15 & 14,6 \\
\hline Empresa & 30 & 29,1 \\
\hline Privada & 25 & 24,3 \\
\hline Negocio propio & 7 & 6,8 \\
\hline Otro & 8 & 7,8 \\
\hline Total & 103 & 100 \\
\hline \multicolumn{3}{|c|}{ Antigüedad en la empresa (años) } \\
\hline Uno & 23 & 22,3 \\
\hline Dos & 11 & 10,7 \\
\hline Tres & 20 & 19,4 \\
\hline Cuatro & 4 & 3,9 \\
\hline Cinco & 8 & 7,8 \\
\hline Seis & 8 & 7,8 \\
\hline Siete & 29 & 28,2 \\
\hline Total & 103 & 100 \\
\hline \multicolumn{3}{|c|}{ Puesto } \\
\hline Coordinación & 27 & 26,2 \\
\hline Gerencia & 17 & 16,5 \\
\hline Jefatura & 16 & 15,5 \\
\hline Auxiliar & 16 & 15,5 \\
\hline Otro & 27 & 26,2 \\
\hline Total & 103 & 100 \\
\hline \multicolumn{3}{|c|}{ Ingreso promedio mensual } \\
\hline 1-10.000 pesos & 33 & 32 \\
\hline $\begin{array}{l}10.001-20.000 \\
\text { pesos }\end{array}$ & 33 & 32 \\
\hline $\begin{array}{l}20.001-30.000 \\
\text { pesos }\end{array}$ & 28 & 27,2 \\
\hline $\begin{array}{l}\text { Más de } 30.000 \\
\text { pesos }\end{array}$ & 9 & 8,8 \\
\hline Total & 103 & 100 \\
\hline \multicolumn{3}{|c|}{ Tiempo de traslado } \\
\hline 30 min o menos & 57 & 55,3 \\
\hline $31 \mathrm{~min}$ a $1 \mathrm{~h}$ & 35 & 34 \\
\hline $\begin{array}{l}\text { De } 1 \text { h a } 1 \text { hy } \\
30 \text { min }\end{array}$ & 11 & 10,7 \\
\hline Total & 103 & 100 \\
\hline
\end{tabular}

Fuente: elaboración propia. 
Tabla 3.Ítems para el análisis factorial

\begin{tabular}{|c|c|c|c|}
\hline Pregunta & Descripción & Promedio & $\begin{array}{l}\text { Desviación } \\
\text { estándar }\end{array}$ \\
\hline P10 & $\begin{array}{l}\text { La dirección de mi organización está sensibilizada respecto al } \\
\text { equilibrio entre el trabajo y la vida personal. }\end{array}$ & 3,782 & 1,60 \\
\hline P11 & $\begin{array}{l}\text { Los directivos son un ejemplo para el resto de los empleados a } \\
\text { la hora de ser familiarmente responsables. }\end{array}$ & 3,739 & 1,45 \\
\hline P12 & $\begin{array}{l}\text { Los directivos y empleados de la organización utilizan políticas y } \\
\text { prácticas de conciliación con el personal. }\end{array}$ & 3,673 & 1,28 \\
\hline P13 & $\begin{array}{l}\text { El equilibrio entre trabajo y familia se ha incorporado en la } \\
\text { comunicación externa de la institución donde labora. }\end{array}$ & 3,565 & 1,25 \\
\hline P14 & $\begin{array}{l}\text { Es mi responsabilidad utilizar de forma correcta los beneficios } \\
\text { del balance laboral y familiar. }\end{array}$ & 4,413 & 1,17 \\
\hline P15 & $\begin{array}{l}\text { Tengo apoyo de la organización para poder resolver una situa- } \\
\text { ción personal. }\end{array}$ & 4,065 & 1,09 \\
\hline P16 & Al buscar un nuevo empleo, la flexibilidad es un factor decisivo. & 3,978 & 0,99 \\
\hline P17 & $\begin{array}{l}\text { Tanto los hombres como las mujeres deben tratar de encontrar } \\
\text { un equilibrio entre la familia y el trabajo. }\end{array}$ & 4,847 & 0,88 \\
\hline P18 & No me siento estresada/o en el trabajo. & 3,521 & 0,76 \\
\hline P19 & $\begin{array}{l}\text { Usualmente estoy al tanto de mis compromisos personales, aun } \\
\text { cuando tengo mucho trabajo. }\end{array}$ & 4,065 & 0,76 \\
\hline P20 & $\begin{array}{l}\text { Se destina presupuesto de la compañía para el desarrollo de } \\
\text { políticas/prácticas de beneficio entre la vida laboral y familiar. }\end{array}$ & 3,239 & 0,68 \\
\hline P21 & $\begin{array}{l}\text { La organización debe obtener beneficios fiscales derivados de } \\
\text { las prácticas de balance entre la vida laboral y personal. }\end{array}$ & 3,717 & 0,66 \\
\hline P22 & $\begin{array}{l}\text { El trabajo me permite darle mejores posibilidades (económicas, } \\
\text { educativas, sociales) a mi familia. }\end{array}$ & 4,239 & 0,58 \\
\hline P23 & $\begin{array}{l}\text { La } 3 \text { donde laboro paga los impuestos correspondientes de } \\
\text { cada mes. }\end{array}$ & 4,500 & 0,39 \\
\hline P24 & $\begin{array}{l}\text { Existe una falta de incentivos a las } 3 \text { s para mejorar la situación } \\
\text { laboral de sus empleados. }\end{array}$ & 3,543 & 0,37 \\
\hline P25 & $\begin{array}{l}\text { La organización promueve políticas familiarmente responsables } \\
\text { como salir a tiempo a casa. }\end{array}$ & 3,630 & 0,24 \\
\hline
\end{tabular}

Fuente: elaboración propia.

Tras haber realizado los descriptivos de las variables mediante el análisis factorial, se llevó a cabo también el análisis de la varianza y se encontró el porcentaje de varianza explicada y el valor correspondiente a cada componente. Por tanto, se obtuvieron cinco factores con 9,052 como el valor propio del primero de los componentes, además de reflejarse un 36,207\% de la varianza explicada. El segundo componente presentó un valor propio de 2,929 y 11,717\% como porcentaje de varianza explicada. En resumen, se concluye que un 68,6\% de la varianza acumulada de los cinco componentes es representado con esta prueba.

Por otro lado, la tabla 4 muestra las matrices inicial y rotada. La matriz de 
factores rotados incluye la asociación de las preguntas de P1 a P5 al primer factor, con cargas factoriales mayores de 0,85 . Al segundo factor se le asocian las preguntas de P6 a P10, con una carga factorial mayor de 0,50. Al tercero se le asocian las preguntas de P11 a P15, y así sucesivamente para el resto de los componentes.

\section{Discusión de los resultados}

De manera general, los resultados que se muestran señalan que la may- oría de los encuestados que laboran en el municipio de Monterrey en Nuevo León decide que el rubro más relevante para tener un balance entre trabajo y familia es la responsabilidad social empresarial (RSE), seguido de políticas públicas, flexibilidad de horario, estrés e incentivos fiscales. Además, con base en la estadística mostrada, se determinó la agrupación de las preguntas en los diferentes factores, a partir de las cargas derivadas del análisis factorial.

Tabla 4. Matrices de componentes rotados

\begin{tabular}{|c|c|c|c|c|c|}
\hline \multirow{2}{*}{ Variables } & \multicolumn{5}{|c|}{ Componentes } \\
\hline & RSE & Estrés & Incentivos fiscales & Políticas públicas & Flexibilidad de horario \\
\hline P1 & 0,866 & & & & \\
\hline P2 & 0,807 & & & & \\
\hline P3 & 0,805 & & & & \\
\hline P4 & 0,891 & & & & \\
\hline P5 & 0,575 & & & & \\
\hline P6 & & 0,658 & & & \\
\hline P7 & & 0,857 & & & \\
\hline P8 & & 0,558 & & & \\
\hline P9 & & 0,620 & & & \\
\hline P10 & & 0,511 & & & \\
\hline P11 & & & 0,665 & & \\
\hline P12 & & & 0,585 & & \\
\hline P13 & & & 0,713 & & \\
\hline P14 & & & 0,714 & & \\
\hline P15 & & & 0,536 & & \\
\hline P16 & & & & 0,835 & \\
\hline P17 & & & & 0,886 & \\
\hline P18 & & & & 0,536 & \\
\hline P19 & & & & 0,864 & \\
\hline P20 & & & & 0,738 & \\
\hline P21 & & & & & 0,590 \\
\hline P22 & & & & & 0,721 \\
\hline P23 & & & & & 0,854 \\
\hline P24 & & & & & 0,726 \\
\hline P25 & & & & & 0,523 \\
\hline
\end{tabular}

RSE: responsabilidad social empresarial.

Fuente: elaboración propia con la corrida en el SPSS v. 24. 
Con respecto a la RSE, autores como Panwar et al. (2006) y Dahlsrud (2008) la definen como una serie de políticas que se adoptan voluntariamente por las organizaciones hacia la contribución de los grupos de interés involucrados. Es por ello por lo que se resalta el balance laboral y familiar para atender al grupo de empleados que cuentan con intereses y necesidades significativas para el buen desempeño de las organizaciones. En dicho sentido, existen otras certificaciones como la que otorga el Centro Mexicano de la Filantropía (CEMEFI) para reconocer a las organizaciones que incluyan acciones y políticas de responsabilidad social.

En cuanto a la política pública, es necesario dar a conocer y llevar a la realidad herramientas que les permitan a los trabajadores resolver situaciones que generen estrés laboral y les impidan tener un buen desempeño en sus actividades. Algunas buenas prácticas pueden ser: horarios escalonados, respetar los horarios de comida de los empleados, llegar a tiempo al centro de trabajo, no realizar reuniones fuera del horario laboral, entre otras. Esto tiene la intención de permitir a los integrantes del equipo de trabajo dar tiempo para su profesionalización y a los padres de familia un pleno desarrollo en la crianza de sus hijos. La norma mexicana NOM 035 de la Secretaría del Trabajo y Previsión Social (STPS), la cual tiene como objetivos identificar, analizar y prevenir los riesgos psicosociales y promover entornos favorables en los centros de trabajo, es un ejemp- lo de políticas públicas encaminadas al balance trabajo-familia (Diario Oficial de la Federación, 2018). Existe otra herramienta por parte del gobierno federal para la promoción de la conciliación laboral y familiar, la cual consiste en un distintivo que otorga la STPS, Ilamado distintivo empresa familiarmente responsable, mediante el cual los centros de trabajo acreditan a las empresas como promotores de buenas prácticas laborales, para la óptima implementación de políticas laborales que apoyen la erradicación de la discriminación, la no violencia laboral y contribuyan a poner un alto al hostigamiento sexual. Gracias a este distintivo se ha permitido tener el apoyo de empresas y su contribución voluntaria para el incremento de la productividad y la mejora competitiva (Secretaría del Trabajo y Previsión Social, 2018).

Con respecto al trabajo flexible, según Fleetwood (2007), dichas prácticas afectan de manera directa al balance laboral y familiar. Cabe mencionar que algunas prácticas son para beneficio del empleado y otras para beneficio del empleador. Más aún, las prácticas para beneficio del empleador restringen el balance laboral y familiar. Stock et al. (2014) argumentan que, incluso cuando se considera que en las economías desarrolladas se han reducido las horas de trabajo, dichas horas han presentado un aumento que equivale hasta un mes adicional de trabajo. Stock et al. (2014) también señalaron que el incremento en la cantidad de horas que trabajan las mujeres es hasta tres veces superior 
a las de su contraparte masculina. Por otro lado, White et al. (2003) indican que la presión desde la dirección en las organizaciones lleva al incremento de horas de trabajo para un mayor compromiso y desempeño de los colaboradores. Así mismo, resaltan que las exigencias en el trabajo producen un efecto negativo en las horas laboradas, que deriva en el incremento de trabajo en casa y la carga laboral (Torres-Flórez, 2019).

Finalmente, con respecto a la variable de estrés laboral, derivado de la implementación de una política de balance trabajo-familia se obtienen beneficios tales como reducción del estrés, mayor satisfacción en el matrimonio, relaciones familiares más sanas, autoestima más alta y mayor satisfacción profesional (Boyer et al., 2005).

Ciertamente, el caso expuesto forma parte de la trayectoria para la implementación de una nueva cultura laboral para muchos centros de trabajo, como puede ser el trabajo por cumplimiento de objetivos. De igual manera permite ser referente e identificar retos y buenas prácticas a los que se enfrentan todos los días padres de familia, mujeres, estudiantes, hijos y adultos mayores, ya que la conciliación familiar y laboral implica uno de los principales desafíos para la sociedad mexicana, como son las interminables jornadas laborales, lo cual trae como consecuencia un desequilibrio en las tareas de corresponsabilidad familiar.

Actualmente, pese a la sensibilización e implementación de políticas flexibles, se siguen identificando retos que deben enfrentarse, como pueden ser tener las herramientas de trabajo necesarias para poder realizar un eficaz desempeño laboral, ya que se puede tener el ánimo y la voluntad para otorgar flexibilidad en el desempeño de actividades laborales desde un lugar diferente al centro de trabajo. Sin embargo, ante la falta de herramientas tecnológicas o de conexión, resulta casi imposible el poder superar las barreras de infraestructura en muchos de los países emergentes.

\section{Conclusiones}

En este artículo se presentó un análisis inferencial con datos recabados en el municipio de Monterrey, México, para el año 2018. Conforme a la pregunta principal de este trabajo, ¿qué determinantes inciden en la consecución de un balance laboral y familiar en el personal de las empresas de Monterrey, Nuevo León?, se encuentra que el determinante principal que permite un balance entre trabajo y familia es la responsabilidad social empresarial (RSE) que desarrolla la empresa.

Otro dato interesante encontrado es que si los empleadores disminuyeran el estrés laboral de sus trabajadores, esto impactaría significativamente en el comportamiento laboral y familiar. Se reafirma que las empresas se comprometen a implementar prácticas que le den equilibrio entre el trabajo y la vida personal a los empleados, además de brindar apoyo cuando estos expresen alguna problemática. Por su parte, los empleados han considerado que es relevante contar con 
flexibilidad de horario al momento de buscar una nueva oportunidad laboral.

Además, en este estudio se encontró que la aplicación oportuna de incentivos fiscales por parte del gobierno en las empresas traería un mayor beneficio para estas. Esto se refleja en un mayor interés por incluir las prácticas de balance laboral y familiar, lo que impacta positivamente en la calidad de vida de los trabajadores.

Otro de los hallazgos comprobados es que las empresas que aplican el concepto de responsabilidad familiar son más receptivas a implementar políticas de balance laboral y familiar y que sus directivos son empáticos en las problemáticas que puedan presentar los empleados. Según Chan et al. (2016), cuando los empleados consideran que cuentan con un balance entre las experiencias positivas de su rol en el trabajo y en la vida familiar, estos experimentan satisfacción en ambos roles. Asimismo, los trabajadores son responsables al momento de tener beneficios adicionales a los indicados por la ley, como son los del balance laboral y familiar.

\section{Referencias}

Allen, T. (2001). Family-Supportive Work Environments: The Role of Organizational Perceptions. Journal of Vocational Behavior, 58(3), 414-435. https:// bit.ly/3mzpwLA

Boyer, S. L., Maertz, C. P., Mosley, D. C., Carr, J. C., \& Keough, S. (2003). Work-family conflict: The impact of moderators on the demand-conflict relationship. Academy of Management Meeting.
Brough, P., \& O'Driscoll, M. P. (2010). Organizational interventions for balancing work and home demands: An overview. Work \& Stress, 24, 280-297. https://bit.ly/2JKH6xP

Chan, X. W., Kalliath, T., Brough, P., Siu, O. L., O'Driscoll, M. P., \& Timms, C. (2016). Work-family enrichment and satisfaction: The mediating role of self-efficacy and work-life balance. The International Journal of Human Resource Management, 27(15), 1755-1776. https:// bit.ly/33GLogG

Chioda, L. (2016). Work and family: Latin American and Caribbean women in search of a new balance. The World Bank.

Dahlsrud, A. (2008). How corporate social responsibility is defined: An analysis of 37 definitions. Corporate Social Responsibility and Environ-mental Management, 15, 1-13. https://bit.ly/3/KzjgQ

Diario Oficial de la Federación (2018). Norma Oficial Mexicana 035. Factores de riesgo psicosocial en el trabajo-identificación, análisis y prevención. https://bit.ly/3qq8x0u

Eby, L. T., Casper, W. J., Lockwood, A., Bordeaux, C., \& Brinley, A. (2005). Work and family research in IO/OB: Content analysis and review of the literature (1980-2002). Journal of Vocational Behavior, 66, 124-197. https://bit.ly/36DC7WE

Edwards, J. R., \& Rothbard, N. P. (2000). Mechanisms linking work and family: clarifying the relationship between work and family constructs. Academy of Management Review, 25, 178-199. https://bit.ly/3IFoGLW

Felstead, A., Jewson, N., Phizacklea, A., \& Walters, S. (2002). Opportunities to work at home in the context of work- 
life balance. Human Resource Manegement Journal, 12(1), 54-76. https://bit. ly/3oivMrF

Fleetwood, S. (2007). Why work-life balance now? International Journal of Human Resource Management, 18(3), 387-400. https://bit.ly/2JHJsxi

Frone, M. R., \& Yardley, J. K. (1996). Workplace family-supportive programmes: Predictors of employed parents' importance ratings. Journal of Occupational and Organizational Psychology, 69(4), 351-366.

Gallup. (2017). State of the global workplace. https://bit.ly/3|xlpxe

Greenhaus, J. H., Collins, K. M., \& Shaw, J. D. (2003). The relation between work-family balance and quality of life. Journal of Vocational Behavior, 63, 510-531. https://bit.ly/217w0ST

Guest, D. E. (2002). Perspectives on the study of work-life balance. Social Science Information, 41(2), 255-279. https://bit.ly/39LliMp

Haar, J. M. (2013). Testing a new measure of work-life balance: A study of parent and non-parent employees from New Zealand. The International Journal of Human Resource Management, 24, 3305-3324. https://bit.ly/33HoYMc

Hirschi, A., Shockley, K. M., \& Zacher, H. (2019). Achieving work-family balance: An action regulation model. Academy of Management Review, 44(1), 150171. https://bit.ly/3mQEixu

Howson, M. \& O'Driscoll, M. P. (1996). Satisfaction with childcare and job-family conflict: Implications for absenteeism lateness, and turnover intentions [tesis no publicada, Universidad de Waikato, Hamilton, Nueva Zelanda].
Jiménez, B. M., Vergel, A. I. S., Muñoz, A. R., \& Geurts, S. A. E. (2009). Propiedades psicométricas de la versión española del cuestionario de interacción trabajo-familia (SWING). Psicothema, 21(2), 331-337. https://hdl.handle. net/2066/76872

Leung, Y. K., Mukerjee, J., \& Thurik, R. (2020). The role of family support in work-family balance and subjective well-being of SME owners. Journal of Small Business Management, 58(1), 130-163. https://bit.ly/3ophxB8

Marks, S. R., \& MacDermid, S. M. (1996). Multiple roles and the self: a theory of role balance. Journal of Marriage and the Family, 58(2), 417-432. https://bit. ly/2leQqJW

Organización de las Naciones Unidas. (2020). Objetivos de desarrollo sostenible. https://bit.ly/3IDqTb0

Panwar, R., Rinne, T., Hansen, E., \& Juslin, H. (2006). Corporate responsibility: Balancing economic, environmental, and social issues in the forest products industry. Forest Products Journal, 56 (2), 4-12. https://bit.ly/3g9qcVO

Secretaría del Trabajo y Previsión Social. (2018). Modelo de Reconocimiento de Empresa Socialmente Reponsable. https://bit.ly/36DHVkN

Sirgy, M. J., \& Lee, D. J. (2018). Work-life balance: An integrative review. Applied Research in Quality of Life, 13(1), 229254. https://bit.ly/3ojTVOY

Stock, R., Bauer, E.-M., \& Bieling, G. (2014). How do top executives handle their work and family life? A taxonomy of top executives' work-family balance. The International Journal of Human Resource Management, 25(13), 1815 1840. https://bit.ly/39CP1 rO 
Torres-Flórez, D. (2019). Estrategia de compensaciones como herramienta de satisfacción laboral. Revista GEON (Gestión, Organizaciones Y Negocios), 6(2), 4-9. https://doi. org/10.22579/23463910.181
White, M., Hill, S., McGovern, P., Mills, C., \& Smeaton, D. (2003). 'High-performance'management practices, working hours and work-life balance. British Journal of Industrial Relations, 41(2), 175-195. https://bit.ly/3|BYMs| 